\title{
Embedded Adaptive Self-Tuning Control Development by a Free Toolchain
}

\author{
Gernot Grabmair*, Simon Mayr \\ Upper Austria University of Applied Sciences, Stelzhamerstrae 23, 4600 Wels, Austria
}

Copyright (C) 2015 by authors, all rights reserved. Authors agree that this article remains permanently

open access under the terms of the Creative Commons Attribution License 4.0 International License

\begin{abstract}
In this study, we present an adaptive self-tuning controller (STC) design for small embedded systems. The new free tool-chain for model based control design is based, among other software, on the open simulator Scilab-XCos. After a very short introduction of model based design terms, this article focuses on the code generator and the other programs of the tool-chain. The design concept is demonstrated by the non-trivial adaptive self-tuning control (STC) of the cart system in simulation and on a real laboratory experiment.
\end{abstract}

Keywords Open Source, Code Generation, Scilab-XCos, Model Based Control, Adaptive Control, Embedded Systems

\section{Introduction}

Model-Based Design (MBD) is a mathematical and visual method of addressing problems associated with complex control design, signal processing and communication systems. It is used in many motion control, industrial equipment, aerospace, and automotive applications. Model-based design is a methodology applied in designing embedded software. During the past years there is a growing interest of medium to small size engineering companies in order to cut down development time and costs. Due to the origin of MBD in aerospace and automotive industries, common commercial tool-chains are quite expensive.

Commercial code generators for Matlab-Simulink (M\&S) - one of the most complete tool-chains in MBD, Dymola, etc. do exist. On the other hand, INRIA and others provide free code generators for the outdated Scilab-Scicos - an open source pendant of M\&S, e.g., [2], [3], and some more. Scilab-XCos [1] made a major development step concerning the user interface in the last two years, but unfortunately the former free code generators do not work anymore. To the best knowledge of the authors there is only one commercial implementation for the new Scilab-XCos suitable for embedded systems.

The main idea presented in this paper is the MBD development using a complete free (or low-cost if target hardware is included) tool-chain starting from the modeling and control design to the hardware realization using an integrated devel-

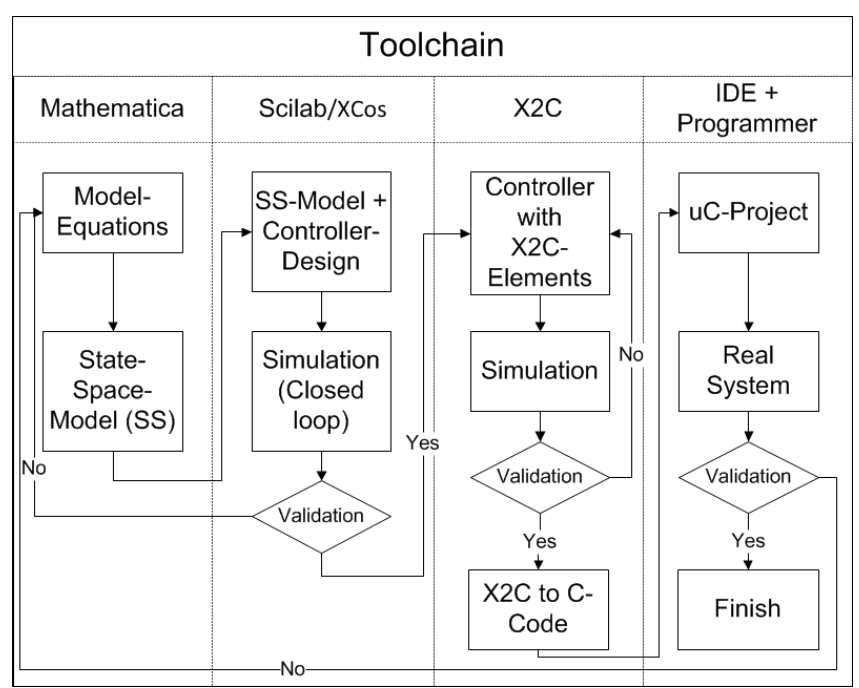

Figure 1. MBD and the tool-chain.

opment environment (IDE). Possible fields of application for such a low-cost development tool-chain are teaching courses and companies interested in testing this new technology or dealing with MBD projects of moderate complexity.

Our contribution includes the overall concept of the toolchain, the interaction between the different free software packages, the know-how relating to prameter identification and adaptive self-tuning control and the implementation on the STM32F4 $\mu \mathrm{C}$ board.

This paper is organized as follows. Starting with a short description of the parts of the whole tool-chain we focus on the details of the code-generator itself. Afterwards, an adaptive control law for the cart system is derived as a nontrivial application. Finally, the model based design process is demonstrated by the implementation of this controller on a low-cost embedded system board.

\section{MBD - the tool-chain}

For the major development steps of a model-based controller design (plant modeling, control design and simulation, code generation and transfer to the target) the reader is referred to Fig. 1. The plant modeling and control design steps are already possible in the standard Scilab-XCos. Scilab is 
a free and open source software for numerical computation maintained by Scilab Enterprises, similar to the commercial Matlab from Mathworks. The graphical dynamical system modeler in Scilab is called XCos and may be seen as the counterpart to Simulink from Mathworks. Its range of features can be extended via add-ons. For example there exists an add-on called "plotting library" that helps the user to make plots with a similar syntax as in Matlab. All plots in this paper are done in Scilab. From an engineering point of view, the out-of-the-box incorporation of the Modelica based Coselica (see [8]) is a valuable tool for designers used for standard electrical and mechanical system blocks. System identification and simulation verification is done in Scilab-XCos too. At least for more dedicated control laws the system has to be described in mathematical terms, i.e., in the form of ODEs. Usually, this is achieved in Mathematica, Maxima or Sage via the Lagrange formalism. Most systems allow the export of equations in a Matlab or $\mathrm{C}$ like syntax that can be included into XCos without effort. Of course, these ODEs can be used as plant model too, alternatively to Coselica.

After the controller has been developed and tested in the simulation environment, it must be converted into C-code and transferred to the target. This process should occur as automated as possible. There are several possibilities to generate $\mathrm{C}$-code from an existing XCos schematic. In order to name a few:

- Gene-Auto, [3]: The Gene-Auto project has created an open-source tool set which converts an application described in a high-level modeling language like Scicos to C-code. Unfortunately, this tool - apart from a commercialized toolbox - only works with the previous version of XCos, called Scicos.

- Real-time Linux as target system, [2]: It is one of the longer known tool-chains, but it supports the outdated Scicos and is not really applicable to small embedded systems, i.e. microcontrollers.

- Scicos-FLEX, [4]: It is somehow a port of [2] for some microcontrollers, but outdated too.

As alternative, we make use of our own generator X2C - see below for a more detailed description. Depending on the target, for ARM Cortex-M devices the free emIDE, see [9], is used. There, a general hardware project with the input-output mapping for the STM32F4-discovery board (approx. 15\$) has been implemented that directly includes the generated controller code. In order to transfer the program to the target, a SEGGER JLink-EDU (approx. 50\$) is used. Nevertheless, it can be replaced by a free JTAG device, e.g., [10]. Alternatively, there is ongoing development in order to establish an industrial control system (PLC from Bernecker and Rainer, b\&r) as an industrial target.

\subsection{The code generator $\mathrm{X} 2 \mathrm{C}$}

The code generator $\mathrm{X} 2 \mathrm{C}$, see [5], was originally developed more than ten years ago at the JK-University Linz, Austria as a Simulink extension generating assembler code for TIDSPs. Later, the system was extended to generate C-code and to largely comply with MISRA (S2C), see [7]. This long history and the simple effective concept of the code generator system stands for stability of its main kernel.

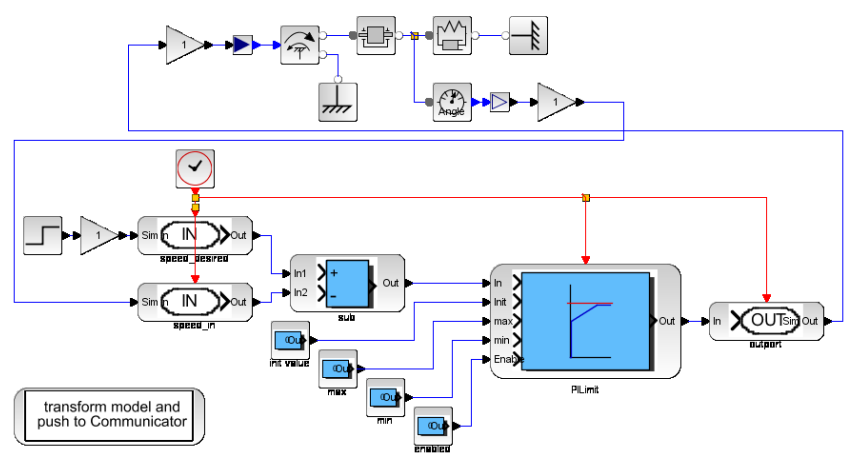

Figure 2. Typical system model with plant (top) and controller (bottom).

$\mathrm{X} 2 \mathrm{C}$ natively includes into XCos and can be simulated in parallel with plant and the controller, see Fig. 2. For the plant, blocks of the XCos Coselica library are used. The controller part is modeled using dedicated X2C-XCos blocks. These blocks are full featured XCos blocks extended with an enhanced parameter editor and the connection to the backend for, e.g., code generation. All the glue code needed for these $\mathrm{X} 2 \mathrm{C}-\mathrm{XCos}$ blocks is fully auto generated from the $\mathrm{X} 2 \mathrm{C}$ block's model. It is possible and also available to automatically generate blocks for other simulation environments. Special blocks are used to model and specify the targets in-ports and out-ports which may correspond to, e.g., analog, digital or PWM input or output ports (IOs).

This system model will be simulated to provide detailed feedback about the expected performance of the overall system for further optimization. In this simulation the blocks implement exactly the code which will run on the target. Thus, effects like quantization, fixed point arithmetic artifacts and discretisation are included in the simulation model. In simulation the developer has easy access to all signals of the plant and controller to analyse the behaviour or to inject faults for testing.

To move on to the target implementation the model transformation and code generation is executed by a single mouse click. The model transformation will step through the XCos model ignoring all non-X2C-XCos blocks to detect all X2C$\mathrm{XCos}$ blocks and their associated clock domain and hence the sample time. X2C features the use of multiple sample times for blocks. The result is an abstract model implemented in Java, holding all relevant information about the blocks, their parameters and their connections. Further on, the code generator is applied to this abstract model using methods from graph theory to check, to partition, to order the model and to generate the final code. The generated code is written as substantially MISRA [7] conform ANSI C code in object oriented style. During this process the parameters specified in the blocks, usually in the continuous time or frequency domain, are automatically converted to implementation specific discrete time domain parameters.

During designing the code generator attention has been paid on generation of human friendly, readable code. The generated code facilitates code review, debugging and potential long-term maintenance.

The central tool for the developer is the so-called Communicator, see Fig. 3. The Communicator is the home of the code generator (fixed-point, single and double data typing is available) and the interface to the modeling / simulation environment and the target. An optional part of $\mathrm{X} 2 \mathrm{C}$ is a quite basic operating system for various target platforms. 


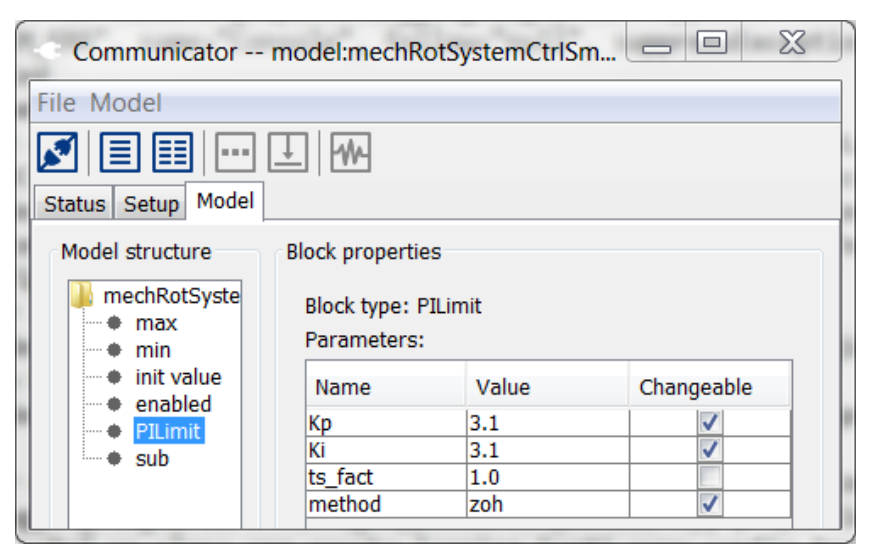

Figure 3. The X2C Communicator with loaded model showing block's parameter.

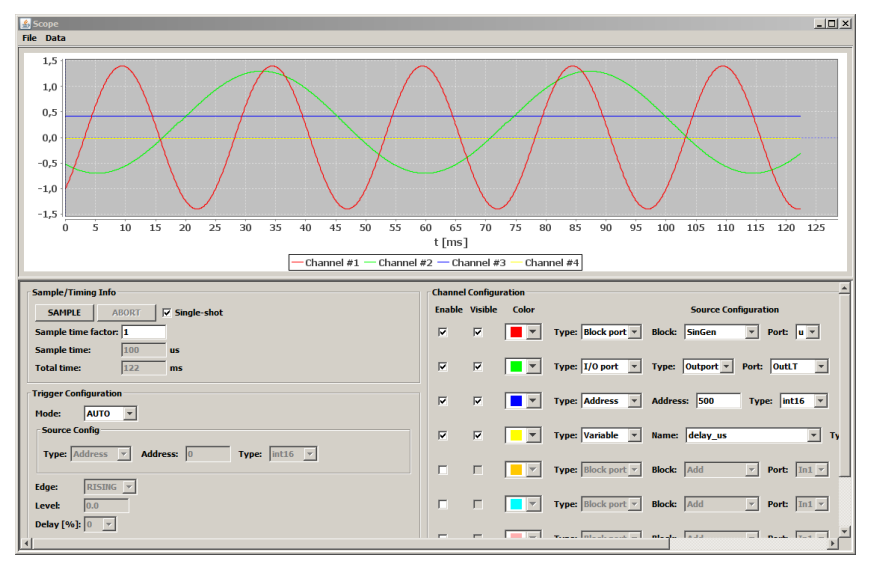

Figure 4. X2C Scope: online access to IO ports, block ports, variables and registers of the target.

This operating system features the communication protocol to connect the Communicator, a flash algorithm to deploy the developed control software, to change parameters online, to record data and more. In the case of applying the whole operating system, there is no specific target IDE or hardware programmer, besides the target compiler, necessary because the host-target link is established by standard serial (UART, USB) or network connections.

For effective development rapid feedback on changes in design is valuable. With $\mathrm{X} 2 \mathrm{C}$ the way from an adapted model to a running system on the target is short even when the whole tool chain of code generator and compiler has to be applied. As a highlight of $\mathrm{X} 2 \mathrm{C}$ this is not necessary in many cases. With X2C parameters can be changed in the model or in the Communicator and the parameters on the target are updated instantly. That means manually tuning controller parameters becomes a task of selecting the block and parameter in the XCos diagram and using the keyboard or mouse wheel to tune the parameter while watching the plant and the automatically updated Scope for feedback. The Scope, see Fig. 4, is featuring functionality like a conventional oscilloscope. Through the Scope the developer has access to online data of block ports, I/O ports, variables and registers of the target in a way like in the simulation environment.

\subsubsection{User defined blocks}

Own code fragments can be incorporated into simulation and code generation with the help of a dedicated block generator. The user specifies the in- and outputs of the block and

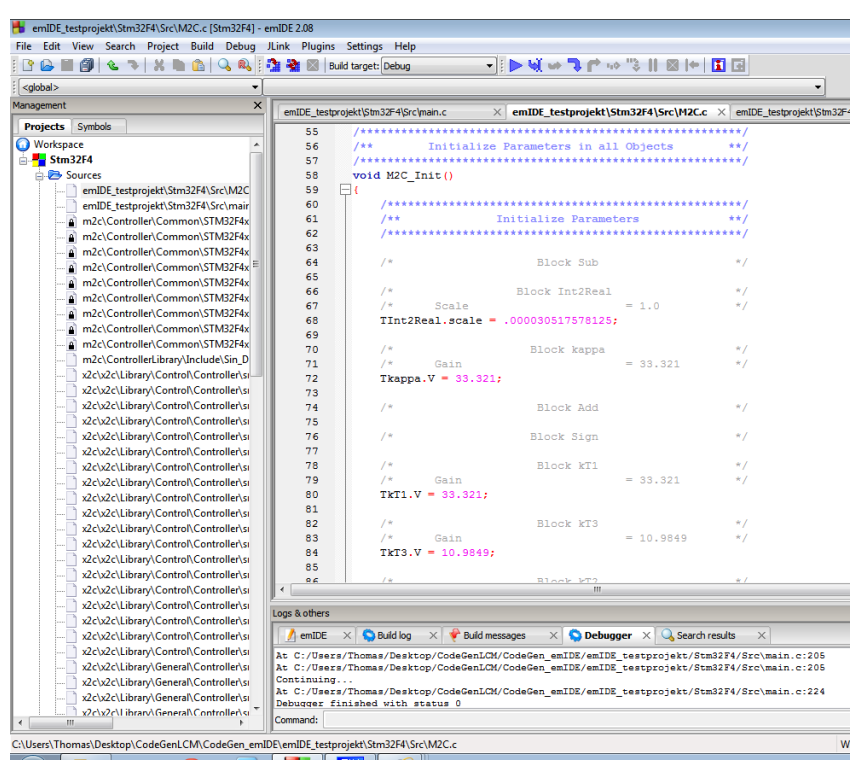

Figure 5. The emIDE with the generated initialization code.

the block generator generates the necessary commented template files afterwards. The behaviour of the block is included into the templates by the user, and can be used for simulation and implementation. In the demonstration application this feature is applied in order to implement a recursive least square algorithm for the adaptive controller.

\section{2 emIDE and hardware programming}

Most microcontroller developers require full insight into the target programming and a JTAG hardware connection for full on-chip debugging features instead of the small operating system approach already mentioned. Towards this end, we use a free microcontroller IDE, see [9], that supports a vast number of targets. emIDE is a full-featured free embedded IDE with stack, register, etc. views and a number of supported JTAG interfaces. In Fig. 5 the initialization part of the heavy commented auto-generated code in emIDE is presented. As soon as standardized names for block IOs in $\mathrm{XCos}$ are used only one general template project is required per target, where the hardware specific code parts and especially the input-output mapping take place. As soon as this is achieved for a specific target the user just needs to press the compile and program button. As already mentioned, the small operating system can be used alternatively.

\subsection{Different Targets}

As for any other code generator the user acceptance relies, among other things, essentially on the number of supported targets. This is the reason why we favor two target approaches, namely the tight integration by the small operating system approach, best fitted for student use, rapid controller prototyping on the real experiments, and the quite versatile approach where all target hardware specific part is done in the IDE. The tight integration approach requires some more target specific details if a new target is made, but releases the user from target hardware interactions. The second IDEbased approach is very easily extendable to new targets.

At the moment some TI targets and one for a STM32F0/1 do exist using the small operating system approach. For the STM32F4-discovery target we have chosen the free emIDE 


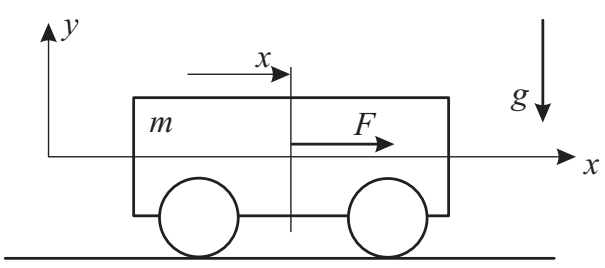

Figure 6. The well-known cart system.

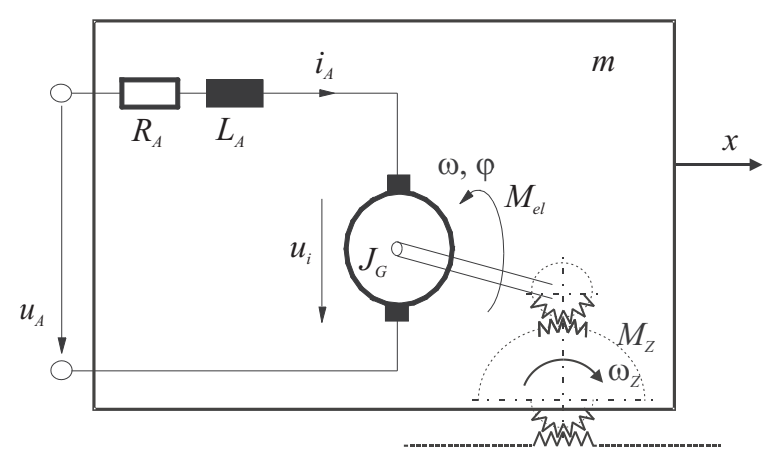

Figure 7. Armature equivalent circuit and power conversion of a dc drive with external excitation.

route for the first time, as the feature rich 32bit Flash CortexM4 based MCU fits general control purposes very well:

- up to $180 \mathrm{MHz} / 225$ DMIPS, with DSP instructions, floating point unit (FPU; single precision) and advanced peripherals

- 2 DAC 12bit, 3x 12bit ADC (24 channels)

- synchronized PWM-timers, quad-encoder channels

- available discovery-board (approx. 15\$)

Towards industrial targets (Programmable Logic Controller, PLC) there is ongoing development concerning the application to a b\&r Powerpanel (PP400) using the IDE approach with the target specific Automation Studio.

\section{Application: cart system}

As a reference application the well-known cart system is presented, see Fig. 6. As common to real world applications the vehicle load capacity is not known. We assume the vehicle mass $\tilde{m}_{1}$, linear friction coefficient $\tilde{d}_{1}$ and static friction coefficient $F_{C}$ as unknown, but constant. The cart with mass $m_{1}$ is driven by a dc drive with external excitation, see Fig. 7.

In the following, we assume a very small electrical time constant $\left(\tau_{e l}=\frac{L_{A}}{R_{A}}\right.$, with armature inductance $L_{A}$ and resistance $R_{A}$ ) compared to the mechanical one. By means of system reduction, i.e, $L_{A} \rightarrow 0$, we introduce equivalent parameters integrating the whole drive-train (drive constant $k_{m}$, inertia $J_{A}$, transmission ratio $n$, gear pinion radius $r$, and some mechanical damping $d_{1}$ ) into the mathematical model of the cart. Other essential parameters are explained in Tab 1.

$$
\begin{gathered}
\tilde{m}_{1}=m_{1}+J_{A}\left(\frac{n}{r}\right)^{2} \\
\tilde{d}_{1}=d_{1}+\frac{n^{2} k_{m}^{2}}{r^{2} R_{A}}
\end{gathered}
$$

The model equations for the non-sticking state of the "slightly" nonlinear reduced system are written to
Table 1. System parameters.

\begin{tabular}{|c|c|}
\hline Name & Description \\
\hline \hline$m_{1}$ & mass cart \\
\hline$d_{1}$ & linear friction coefficient cart \\
\hline$F_{C}$ & static friction coeff. cart \\
\hline$J_{A}$ & moment of inertia of drivetrain \\
\hline$n$ & transmission ratio \\
\hline$k_{m}$ & radius pinion \\
\hline$r$ & motor constant \\
\hline$R_{A}$ & terminal resistance \\
\hline
\end{tabular}

error caused by discrete-filtering of sign-function
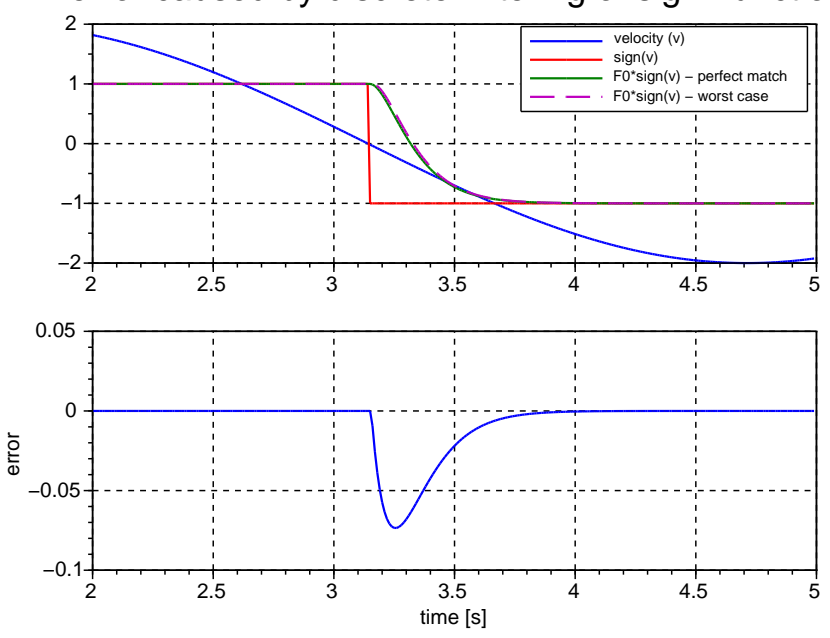

Figure 8. Worst case approximation error for $\operatorname{sign}(v)$ for $T_{s}=10 \mathrm{~ms}$.

$$
\left(\begin{array}{c}
\dot{x} \\
\dot{v}
\end{array}\right)=\left(\begin{array}{c}
v \\
\frac{1}{\tilde{m}_{1}}\left(\beta u_{A}-\tilde{d}_{1} v-F_{C} \operatorname{sign}(v)\right)
\end{array}\right)
$$

with the abbreviation $\beta=n k_{m}\left(r R_{A}\right)^{-1}$ and the static friction coefficent $F_{C}$.

For the calculation of the linear state controller the system has to be written in the form $\dot{\boldsymbol{x}}=\mathbf{A x}+\mathbf{b} u$. We assume that the static friction is ignored because its value will be identified and compensated in all measurements by the well-known approach.

$$
\left(\begin{array}{c}
\dot{x} \\
\dot{v}
\end{array}\right)=\left(\begin{array}{cc}
0 & 1 \\
0 & -\frac{\tilde{d}_{1}}{\tilde{m}_{1}}
\end{array}\right) \cdot\left(\begin{array}{c}
0 \\
\frac{\beta}{\tilde{m}_{1}}
\end{array}\right) \cdot u_{A}
$$

\subsection{Continuous system identification and adaptive con- trol design}

As already mentioned, the cart parameters are assumed unknown but constant. For full physical insight into the adaption parameters, we do not identify the sampled, but the continuous plant itself.

In order to get rid of the time derivatives the second equation of the linear system model is transformed from the time domain to the frequency domain. For brevity, initial states are assumed equal to zero.

$$
s^{2} \tilde{m}_{1} \hat{x}=\beta \hat{u}_{A}-\tilde{d}_{1} s \hat{x}-F_{C} \operatorname{sign}(v)
$$

Some attention has to be paid to the static friction term. Here, we assume a quite small number of switchings so that the Laplace transform exists. Later on we may approximate 

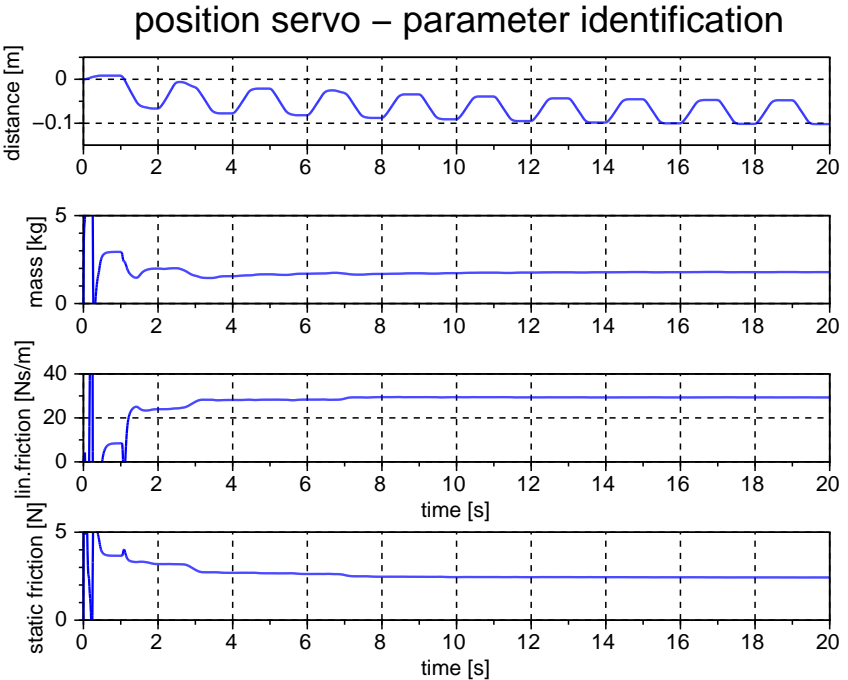

Figure 9. Offline parameter identification with RLS in Scilab-XCos on true measurements.

$\operatorname{sign}(v)$ in a reasonable way. We apply the realizable stable filter with free coefficients $\alpha_{i}$ to both sides

$$
\begin{gathered}
F_{0}(s)=\frac{\alpha_{0}}{s^{2}+\alpha_{1} s+\alpha_{0}} \\
F_{1}(s)=s F_{0}(s)
\end{gathered}
$$

$$
\begin{array}{cl}
\frac{s^{2}}{s^{2}+\alpha_{1} s+\alpha_{0}} \hat{x} \alpha_{0} \tilde{m}_{1}= & \frac{\alpha_{0}}{s^{2}+\alpha_{1} s+\alpha_{0}} \hat{u}_{A} \beta+\quad \text { (7) } \\
-\frac{s}{s^{2}+\alpha_{1} s+\alpha_{0}} \hat{x} \alpha_{0} \tilde{d}_{1} & -\frac{\alpha_{0}}{s^{2}+\alpha_{1} s+\alpha_{0}} \operatorname{sign}(v) F_{C}
\end{array}
$$

see [11]. With standard polynomial division one eliminates the non-strictly proper transfer function. Therefore, it is sufficient to implement the two filters $F_{0}(s)$ and $F_{1}(s)=s F_{0}(s)$. Both filters have a common denominator and can be realized as one dynamical system if applied to the same signal. The inverse Laplace transform leads to one data line of the algebraic equation system for identification

$$
\begin{aligned}
\left(\alpha_{0}\left(x-\frac{\alpha_{1}}{\alpha_{0}} F_{1} * x-F_{0} * x\right)\right. & \left.F_{1} * x \quad F_{0} * \operatorname{sign}(v)\right) \\
& \cdot\left(\begin{array}{c}
\theta_{1} \\
\theta_{2} \\
\theta_{3}
\end{array}\right)=F_{0} * u_{A} \beta
\end{aligned}
$$

linear in the unknown parameters $\theta_{1}=\tilde{m}_{1}, \theta_{2}=\tilde{d}_{1}$ and $\theta_{3}=F_{C}$, whereby $*$ indicates the convolution operator in time-domain. For implementation purpose the filters have to be discretized and $\operatorname{sign}(v)$ approximated. Towards this end, the velocity for $\operatorname{sign}(v)$ is approximated by the backward difference quotient and further the sign-function by a sum of positive and negative discretized unit steps which only causes a quite small error as long as there is no chattering around zero velocity. Additionally, this error vanishes with the arbitrary filter time constants as depicted in Fig. 8.

The filter on plant input $u_{A}$ is discretized naturally by a zero-order hold, but the filters applied to the states have to be approximated. A trapezoidal approximation, i.e., the Tustin method, is usually sufficient without requiring an unnatural small sample time compared to system dynamics. The unknown parameters can be estimated using standard recursive

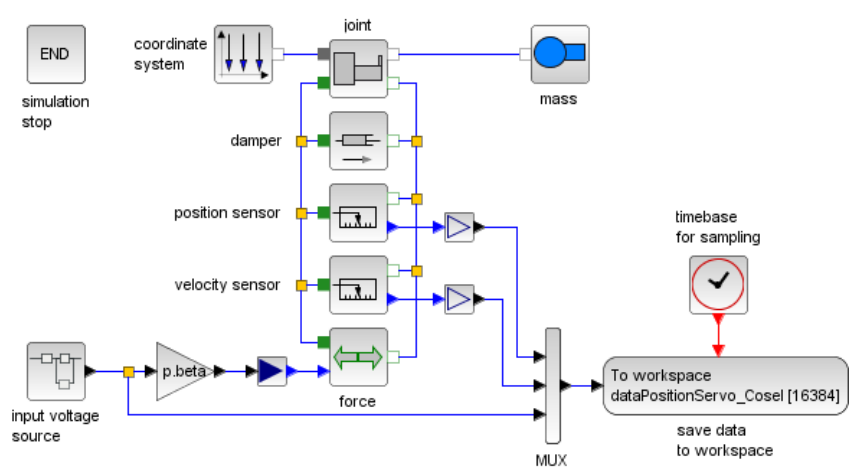

Figure 10. Cart system - Coselica.
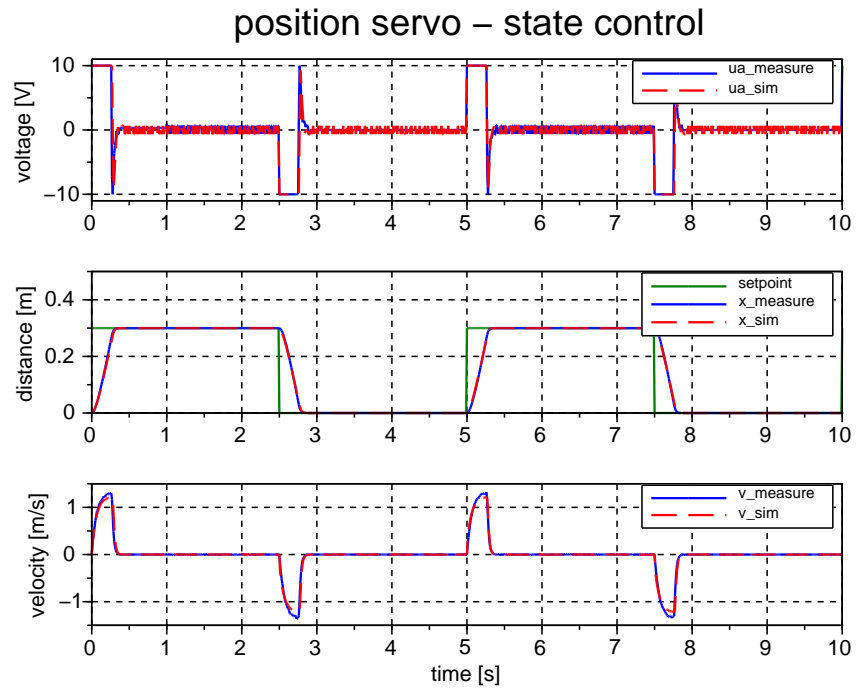

Figure 11. Comparison of simulation and true experiment with standard state control and known parameters.

least square (RLS) algorithm. For identification results with the RLS algorithm in XCos on true plant measurements the reader is kindly referred to Fig. 9. The parameter drift until $8 \mathrm{~s}$ is caused by the unequal mechanical behaviour of the laboratory test bench and the drifting mean value of the position cycles due to the open loop experiment.

For the adaptive self-tuning control (STC) approach the filters and a standard RLS algorithm are implemented in combination with a linear state control law parametrized in $\tilde{m}_{1}$ and $\tilde{d}_{1}$.

The necessary $\mathrm{X} 2 \mathrm{C}-\mathrm{XC}$ os blocks for parameter identification (signal filtering and RLS algorithm) and STC (control law) are coded manually with the help of the block generator.

\subsection{Experiment - Cart system}

In order to demonstrate the physical modeling capabilities the Coselica toolbox is used for plant modeling, see Fig. 10. The quality of the overall model compared to the true experiment can be seen in Fig. 11 where a standard linear quasicontinuous $\left(T_{s}=1 \mathrm{~ms}\right)$ state controller based on fixed identified parameters is implemented in simulation and real experiment. Velocities are obtained by first order derivative approximations.

\subsubsection{Adaptive STC Control}

In order to meet the proposed goal, STC control with unknown, but constant parameters is applied to the real plant. 
position servo - self tuning state control (STC)
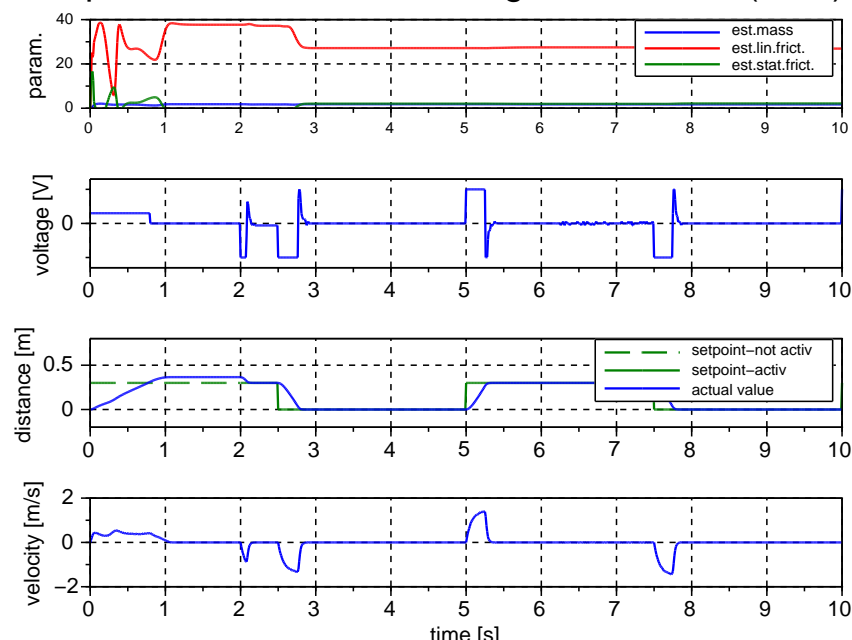

Figure 12. STC control measurement (slashed lines means inactive signals)

The first 2 seconds are used for settling the online RLS identificator and the filters as derived above. Then the parametrized control law $u_{A}=-\mathbf{k}(\boldsymbol{\theta})^{\mathrm{T}} \mathbf{x}$ is activated, see Fig. 12.

$$
\mathbf{k}=\left(\begin{array}{c}
-1576.73 \tilde{m}_{1} \\
0.2594 \tilde{d}_{1}-40.449 \tilde{m}_{1}
\end{array}\right)
$$

\section{Conclusion}

The presented work is available for free - to a large extent under a BSD licence, see the references [5], [6].

Ongoing development is targeted towards efficient handling of vectorized signal lines, Scilab-code to C-code conversion, more block libraries, industrial targets, IEC 611313 code generation (PLC), adaption of the FMI (functional mockup interface) for model exchange with various commercial simulators, and some state machine concept. For most parts we plan to again adapt existing free tools.

\section{Acknowledgements}

We gratefully acknowledge the support from the Austrian funding agency FFG in the COIN-project ProtoFrame and the internal funding in the project MOdoPS. Part of this work was conducted within the research project "sustainable and resource saving electrical drives through high energy and material efficiency" sponsored within the EU program "(Regio 13)" and within the COMET K2 center ACCM.

\section{REFERENCES}

[1] Scilab: Free and Open Source software for numerical computation, Scilab Enterprises, 2012, http://www.scilab.org/.

[2] Roberto Bucher, et al., RTAI-Lab tutorial: Scilab, Comedi, and real-time control, 2006.

[3] Ana-Elena Rugina, et al., Gene-Auto: Automatic Software Code Generation for Real-Time Embedded Systems, DASIA 2008.

[4] Scicos-FLEX code generator, http://erika.tuxfamily.org/drupal/scilabscicos.html/ .

[5] X2C in Scilab-XCos, 2013, http://www.mechatronicsimulation.org/ .

[6] 2013, http://www.modelbaseddesign.at/ .

[7] MISRA Consortium, 2013, http://www.misra.org.uk/.

[8] Reusch, 2013, http://www.kybdr.de/software/.

[9] emIDE the free IDE for embedde programming, 2013, http://www.emide.org/ .

[10] Open On-Chip Debugger, a open JTAG interface, 2013, http://openocd.sourceforge.net/ .

[11] JJE. Slotine, W. Li, Applied nonlinear control, Prantice-Hall, 1991. 